An International Journal of Language, Literature and Gender Studies (LALIGENS), Ethiopia

Vol. 4 (1), Serial No 9, January, 2015:34-45

ISSN: 2225-8604(Print) ISSN 2227-5460 (Online)

DOI: http://dx.doi.org/10.4314/laligens.v4i1.3

\title{
Etudes Comparatives Des Termes Anglais Et Français Relatifs Au Jeu De Football (En Français Et En Anglais)
}

\author{
Asadu, Felicia Oluchukwu, Ph.D. \\ Université Nnamdi Azikiwe \\ Département des Langues Vivantes Européennes \\ E-mail:asaduoluchukwu@yahoo.com \\ Phone No: 08038740016
}

Résumé: La terminologie est l'ensemble des mots qui appartiennent à un champ de science particulière. C'est en d'autre part les techniques de classification, de création de nouveaux termes. Pourtant dans ce travail les termes n'étaient pas créés parce que les deux langues (français-anglais) sont déjà formées. Nous avons fait qu'assembler ces termes pour les rendre spontanément accessible aux commentateurs qui diffusent à la télé pendant le jeu africain des nations sans recourir à leurs dictionnaires bilingues.

Mots clés la terminologie, le football

\begin{abstract}
Terminology is the group of words that belong to a particular field of study. It is on the other hand a science of classification and creation of new words. However in this work no terms were created as the two languages are already developed (EnglishFrench).Terms were simply assembled to make them easily accessible to television commentators with a view to saving them the task of always having recourse to the dictionary for Africa World Cup commentators.
\end{abstract}

Key words: Terminology, Football 


\section{Introduction}

La terminologie est l'ensemble des mots qui appartiennent à un champ de science particulière. C'est en d'autre part les techniques de classification, de création de nouveaux termes. Pourtant dans ce travail on n'a pas créé des termes parce que les deux langues (français-anglais) sont déjà formées. On a fait qu'assembler ces termes pour les rendre spontanément accessible aux commentateurs qui diffusent à la télé pendant le jeu africain des nations sans recourir à leurs dictionnaires bilingues.

Le football est un jeu intéressant. Certains le suivent pour se divertir, les autres pour critiquer les joueurs. Il y en a d'autres qui y assistent pour prendre les techniques différentes, le plus import est pour le spectacle. Quoi que soit le but des spectateurs, le football reste un des jeux populaires au monde. Pour que les spectateurs bilingues profitent de ce jeu, nous avons besoin de compiler les listes de termes en deux langues internationales qui sont l'anglais et le français.

Les anglophones ne savaient pas l'équivalence de ces termes relatifs au football en français tandis que les francophones ne les savaient qu' en français. Le francophone aura la tendance de traduire $<<$ red card $>>$ comme carte rouge au lieu de carton rouge parce qu'il ne sait pas le sens de <red card> en anglais. Ce qu'il a fait dans ce contexte c'est de calquer ces mots au lieu de trouver ces équivalences en français vise versa. Pour que ces gens en profitent nous avons classifié les termes d'une manière que les commentateurs sportifs pourraient les employés spontanément au cours des matchs de football. Nous savons que l'étude comparative est un aspect important de la recherche au cadre théorique et appliqué. Dans cet optique Johansson et Hoffland opinent:

Language comparison is of great interest in a theoritical as well as an applied perspective.It reveals what is general and what is language specific and is therfore important both for the understanding of languages in general and for the study of the individual languages compared.

Glossary of Terms

\begin{tabular}{|l|l|l|}
\hline & Français (contexte definitoire) & English (Defining context) \\
\hline 1. & Amateur & Young Star \\
\hline 2. & Annonceurs & Reporters \\
\hline 3. & Anciens Joueurs & Old players \\
\hline 4. & Attaquants & Attackers \\
\hline 5. & Blessures & Injuries \\
\hline 6. & Buts & Goals \\
\hline
\end{tabular}

Copyright $\odot$ IAARR 2014: $w$ ww.afrrevjo.net/laligens Indexed and Listed by AJOL \& EBSCOhost 


\begin{tabular}{|l|l|l|}
\hline 7. & Champions & Champions \\
\hline 8. & Club & Club \\
\hline 9. & Coach & Coach \\
\hline 10. & Comité executive & Executive Committee \\
\hline 11. & Compétitions & Competitions \\
\hline 12. & Commentateurs & Commentators \\
\hline 13. & Conféderation Africaine de Football & Confederation of African Football \\
\hline 14. & Contrat & Contract \\
\hline 15. & Coupe du monde & World cup \\
\hline 16. & Coups de pied & Kick-off \\
\hline 17. & Corners & Corner \\
\hline 18. & Début & Opening match \\
\hline 19. & Démi-finales & Semi-finals \\
\hline 20. & Défense & Defence \\
\hline 21. & Défenseurs & Defenders \\
\hline 22. & Elimination & Elimination \\
\hline 23. & Entraineur & Coach \\
\hline 24. & Equipe & Team \\
\hline 25. & FIFA & FIFA \\
\hline 26. & Finale & Final \\
\hline 27. & Finalistes & Finalists \\
\hline 28. & Football & Ball \\
\hline 29. & Footballeurs & Footballers \\
\hline 30. & Gardiens de but & Goal Keeper \\
\hline 31. & Jeux Olympiques & Olympique games \\
\hline 32. & Joueurs & Players \\
\hline 33. & Latéraux & Strikers \\
\hline 34. & Légendaire & Legendary \\
\hline 35. & Lions Indomptables & Indomitable Lions \\
\hline 36. & Maîtres entraineurs & Head coach \\
\hline 37. & Match & Match \\
\hline 38. & Medaille d'or & Gold Medal \\
\hline 39. & Milieu de terrain & Mid-fielder \\
\hline 40. & Penalty & Penalty \\
\hline 41. & Première mi-temps & First half \\
\hline 42. & Prolongation & Extension \\
\hline 43. & Quarts de finale & Quarter final \\
\hline 44. & Spectateurs & Spectators \\
\hline & & \\
\hline
\end{tabular}




\begin{tabular}{|l|l|l|}
\hline 45. & Supporteurs & Suporters \\
\hline 46. & Télévisions & Televisions \\
\hline 47. & Trophéé & Trophy \\
\hline 48. & Tournoi & Tournament \\
\hline 49. & Vainqueurs & Winners \\
\hline 50. & Victoire & Victory. \\
\hline
\end{tabular}

Etude comparative des termes français-anglais

\begin{tabular}{|c|c|}
\hline $\begin{array}{l}\text { Terme source source } \\
\text { term/langue de départ }\end{array}$ & $\begin{array}{l}\text { Terme d'arrivée Target term/langue } \\
\text { d'arrivée }\end{array}$ \\
\hline $\begin{array}{l}\text { Amateurs (N. Ref. no. } 1 \text { pg. } 25 \text { ) } \\
\text { Contexte sources (Source context) : Au } \\
\text { plan matériel, les pros Nigérians ou sud- } \\
\text { africains d'aujourd'hui n'ont plus grand- } \\
\text { chose à voir avec les amateurs marocains } \\
\text { ou Zairois d'il y a vingt -Cinq ans. }\end{array}$ & $\begin{array}{l}\text { Young players ( } \mathrm{N}, \text { No. pg. } 20) \text { contexte } \\
\text { d'arrivée (Target context): With the kind of } \\
\text { experience these young players get from } \\
\text { playing with the } 11=20 \mathrm{~s} \text {. }\end{array}$ \\
\hline $\begin{array}{l}\text { Annonceurs ( } \mathrm{N}, \text { No.12 pg. 26) } \\
\text { source (source context) }: \text { Les } \\
\text { annonceurs, desireux d'accroître leur } \\
\text { notoriété sur le contient. }\end{array}$ & $\begin{array}{l}\text { Reporters }(\mathrm{N}, \text { No. } 10 \text { pg. } 18) \text {. contexte } \\
\text { d'arrivée (Target context) : In every big } \\
\text { competition, the hardest part is always the } \\
\text { semi-final the Frenchman told reporters } \\
\text { after the Black Stars training session in } \\
\text { Accra. }\end{array}$ \\
\hline $\begin{array}{l}\text { Attaquants }(\mathrm{N}, \text { No.13 pg. } 10) \text { Contexte } \\
\text { source (source context) : Reproche } \\
\text { Injuste. Tous les attaquants savant bien } \\
\text { que le passer "à la "Régulière" relève de la } \\
\text { gageure. }\end{array}$ & $\begin{array}{l}\text { Attackers (N, No } 12 \text { pg } 14) \text { contexte } \\
\text { d'arrivée: (Target context). The } 25 \text { year old } \\
\text { attacker missed U.S.A } 94 .\end{array}$ \\
\hline $\begin{array}{l}\text { Anciens joueurs ( } \text { N, No.15 pg. } 15) \\
\text { Contexte source (source context) : Tous } \\
\text { les grands footballeurs de Pele a Cruyft, } \\
\text { en passant par chalton ou Maradone. }\end{array}$ & $\begin{array}{l}\text { Old players ( } \mathrm{N}, \text { No. } 11 \mathrm{pg} .20) \text { Contexte } \\
\text { d'arrivée (Target context): The team is } \\
\text { retaining } 18 \text { of its old players. }\end{array}$ \\
\hline $\begin{array}{l}\text { Blessures ( } \mathrm{N}, \text { No. } 14 \text { pg. 20) Contexte } \\
\text { source (source context) : Malgré les } \\
\text { blessures de nombreux titulaires, contre } \\
\text { cette même Allemagne. }\end{array}$ & $\begin{array}{l}\text { Injury (N, No. } 21 \text { pg. 10) contexte } \\
\text { d'arrivée (Target context) : Romario \& } \\
\text { Boksk have succumbed to injury. }\end{array}$ \\
\hline $\begin{array}{l}\text { Buts }(\mathrm{N}, \text { No.1 pg. } 24) \text { Contexte source } \\
\text { (source context) : Deux buts, contre son } \\
\text { camp lors du match contre la France. }\end{array}$ & $\begin{array}{l}\text { Goals (N. No } 22 \text { pg. 24). } \\
\text { contexte d'arrivée (Target context) } \\
\text { Batistuta has scored } 124 \text { goals. }\end{array}$ \\
\hline
\end{tabular}




\begin{tabular}{|c|c|}
\hline $\begin{array}{l}\text { Champions (N, No.15 pg. 25) Contexte } \\
\text { source: (source context) : Or on ne } \\
\text { "Chambre" pas impunément les triples } \\
\text { Champions du monde. }\end{array}$ & $\begin{array}{l}\text { Champions (N, No.1) pg. 15) contexte } \\
\text { d'arrivée (Target context): The Eagles } \\
\text { were going into their match with Bulgaria... } \\
\text { defending African Champions. }\end{array}$ \\
\hline $\begin{array}{l}\text { Club }(\mathrm{N}, \text { No.16 pg. 8) Contexte source : } \\
\text { (source context) : Vous êtes devant le } \\
\text { centre de formation du grand "club" } \\
\text { abidjanais }\end{array}$ & $\begin{array}{l}\text { Club }(\mathrm{N}, \text { No. } 1 \text { pg. } 4) \text { contexte source } \\
\text { (source context): Rufai and Baruwa are } \\
\text { reserved in their clubs. }\end{array}$ \\
\hline $\begin{array}{l}\text { Coach }(\mathrm{N}, \text { No.13 pg. } 3) \text { Contexte source } \\
\text { (source context }): \text { Alex Ferguson, le } \\
\text { coach de Manchester United ne s'y est } \\
\text { trompé }\end{array}$ & $\begin{array}{l}\text { Coach }(\mathrm{N}, \text { No. } 24 \text { pg. } 8) \text { contexte source } \\
\text { (source context): Trapattoni, not Batistuta's } \\
\text { sort of coach. }\end{array}$ \\
\hline $\begin{array}{l}\text { Comité executif }(\underline{N, \text { No. } 12 \text { pg. } 12)}) \\
\text { Contexte source }(\text { source context }): \text { Cette } \\
\text { nomination devra être enternée par le } \\
\text { Comité executif }\end{array}$ & $\begin{array}{l}\text { Executive Committee (N, No. } 24 \text { pg. } 3) \\
\text { contexte source (source context): The } \\
\text { FIFA's Executive Committee particularly } \\
\text { those from the European Football Union... }\end{array}$ \\
\hline $\begin{array}{l}\text { Compétition (N, No. } 17 \text { pg. } 8) \text { Contexte } \\
\text { source (source context) : La compétition } \\
\text { est d'autant plus vive que, depuis } \\
\text { l'accession. }\end{array}$ & $\begin{array}{l}\text { Compeition ( } \mathrm{N}, \text { No. } 2 \text { pg. } 10) \text { contexte } \\
\text { d'arrivée ( Target context): France } 98 \text { is a } \\
\text { competition in which the five African } \\
\text { representatives would be handled with a big } \\
\text { respect by their opponents. }\end{array}$ \\
\hline $\begin{array}{l}\text { Commentateurs }(\underline{\mathrm{N}, \text { No.17 pg. } 7)}) \\
\text { Contexte source }(\text { source context }): \text { Des } \\
\text { duplex avec les commentateurs et la } \\
\text { régie finale. }\end{array}$ & $\begin{array}{l}\text { Commentators ( } \mathrm{N}, \text { No. } 2 \text { pg.15) contexte } \\
\text { d'arrivée ( Target context): The most } \\
\text { talked about in cup before the start of the cup } \\
\text { lived up to biling commentators describing } \\
\text { the most interesting ... since competition } \\
\text { started to the last day. }\end{array}$ \\
\hline $\begin{array}{l}\text { Confédération Africaine de football }(\underline{\mathrm{N}}, \\
\underline{\text { No}} .18 \mathrm{pg} .12) \text { Contexte source (source } \\
\text { context): Invitée par la Confédération } \\
\text { Africaine de Football (CAF) ... }\end{array}$ & $\begin{array}{l}\text { The Confederation of African Football }(\mathrm{N} \text {, } \\
\text { No.9 pg. } 11) \text { contexte d'arrivée ( Target } \\
\text { context): The confederation of African } \\
\text { Football (CAF) placed Zambia as over all } \\
\text { ninth. }\end{array}$ \\
\hline $\begin{array}{l}\text { Contrat (N, No. } 18 \text { pg. } 6 \text { ) Contexte } \\
\text { source (source context): Ses performance } \\
\text { américaines lui valent de décrocher un } \\
\text { contrat avec l' AC. }\end{array}$ & $\begin{array}{l}\text { Contract }(\underline{\mathrm{N}, \text { No.8 pg.23 }}) \text { Keshi's contract } \\
\text { with Togo has expirèd. }\end{array}$ \\
\hline $\begin{array}{l}\text { Coupe du monde (N, No.18 pg.2) } \\
\text { Contexte source (source context) : Le } 9 \\
-0, \text { le plus gros écart de faute dans }\end{array}$ & $\begin{array}{l}\text { World cup (N, No.7 pg.15) History is } \\
\text { replete with stars who came to the World } \\
\text { cup. }\end{array}$ \\
\hline
\end{tabular}




\begin{tabular}{|c|c|}
\hline l'histoire de la coupe du monde. & \\
\hline $\begin{array}{l}\text { Coups de pied }(\mathrm{N}, \text { No.19 pg.4) Contexte } \\
\text { source (source context)... A coups de } \\
\text { pied et de poing. }\end{array}$ & $\begin{array}{l}\text { Kick-off ( } \mathrm{N}, \text { No. } 7 \text { pg.5) contexte } \\
\text { d'arrivée: (Target context). With about } 72 \\
\text { hours to the Kick-off of the World cup... }\end{array}$ \\
\hline $\begin{array}{l}\text { Corners (N, No.1 pg.9) Contexte source } \\
\text { (source context) : Les corners par } \\
\text { exemple seront filmés avec une camera } \\
\text { haute. }\end{array}$ & $\begin{array}{l}\text { Corner (N, Ref no. } 24 \text { pg. } 18 \text { ) Contexte } \\
\text { d'arrivée (Target context): Soon after, the } \\
\text { Nigerian team settled down to business } \\
\text { forcing the first corner in eighth minute. }\end{array}$ \\
\hline $\begin{array}{l}\text { Début de match }(\underline{\mathrm{N}, \text { No.19 } \mathrm{pg} .14}) \\
\text { Contexte source (source context) : Le } \\
\text { début de match des lions est catastrophe }\end{array}$ & $\begin{array}{l}\text { Opening match }(\mathrm{N}, \text { No.6 pg. } 10) \text { contexte } \\
\text { d'arrivée (Target context): Super Eagles } \\
\text { opening match against Bulgaria for years } \\
\text { ago in USA. }\end{array}$ \\
\hline $\begin{array}{l}\text { Demi- finales ( } \mathrm{N}, \text { No.14 pg.10) Contexte } \\
\text { source (source context). Les demi - } \\
\text { finales, le match de classement et } \\
\text { naturellement, la finale... }\end{array}$ & $\begin{array}{l}\text { Semi - finals ( } \mathrm{N}, \text { No. } 3 \mathrm{pg} .21) \text { contexte } \\
\text { d'arrivée (Target context): Egypt meet } \\
\text { Ivory Coast in semi - finals tomorrow. }\end{array}$ \\
\hline $\begin{array}{l}\text { Défense (N, No.14 pg.14) Contexte } \\
\text { source (source context) : En d'autres } \\
\text { termes, il est l'avant-garde de sa défense } \\
\text { et l'arrière - garde de son attaque. }\end{array}$ & $\begin{array}{l}\text { Defence (N, No. } 23 \text { pg. } 10) \text { contexte } \\
\text { d'arrivée (Target context)... Europe's best } \\
\text { sweeper to marshal the defence. }\end{array}$ \\
\hline $\begin{array}{l}\text { Défenseurs ( } \mathrm{N} \text {, No.9 pg.10) Contexte } \\
\text { source (source context) : L'ensemble des } \\
\text { attaquants et défenseurs dans la surface } \\
\text { de réparation. }\end{array}$ & $\begin{array}{l}\text { Defenders }(\mathrm{N}, \text { No. } 1 \mathrm{pg} .23) \text { contexte source } \\
\text { (source context): Good in the air and he } \\
\text { likes to get the ball to feet, turn and run at } \\
\text { defenders. }\end{array}$ \\
\hline $\begin{array}{l}\text { Elimination ( } \mathrm{N}, \text { No.6 pg. } 23 \text { ) Contexte } \\
\text { source (source context)... Une } \\
\text { élimination sans gloire. }\end{array}$ & $\begin{array}{l}\text { Elimination (N, No.15 pg.14) contexte } \\
\text { source (source context) : Nigeria's } \\
\text { elimination at the quarter final... }\end{array}$ \\
\hline $\begin{array}{l}\text { Entraineur }(\underline{N}, \text { No. } 20 \text { pg. } 10) \text { Contexte } \\
\text { source (source context) : D'ailleurs, } \\
\text { l'entraineur Clause Lerory répète à qui } \\
\text { veut... }\end{array}$ & $\begin{array}{l}\text { Coach }(\mathrm{N}, \text { No. } 20 \text { pg.14 }) \text { contexte source } \\
\text { (source context) : His coach had announced } \\
\text { already that the remarkable little centre } \\
\text { forward will make a difference. }\end{array}$ \\
\hline $\begin{array}{l}\text { Equipe ( } \mathrm{N}, \text { No. } 20 \text { pg.14) Contexte } \\
\text { source (source context) : Comme il aurait } \\
\text { aimé être sur le terrain pour animer le jeu } \\
\text { d'une équipe rajeunie. }\end{array}$ & $\begin{array}{l}\text { Team (N, No. } 4 \text { pg. } 16) \text { Contexte source } \\
\text { (source context): We need to have it in this } \\
\text { national team. }\end{array}$ \\
\hline $\begin{array}{l}\text { FIFA }(\underline{N}, \text { No. } 15 \text { pg. } 4) \text { Contexte source } \\
\text { (source context) : La FIFA insite pour }\end{array}$ & $\begin{array}{l}\text { FIFA }(\mathrm{N}, \text { Ref no. } 4 \text { pg. } 14) \text { Contexte source } \\
\text { (source term): FIFA elects new President }\end{array}$ \\
\hline
\end{tabular}




\begin{tabular}{|c|c|}
\hline $\begin{array}{l}\text { que les deux équipes soient traitées } \\
\text { exactement de la même manière par la } \\
\text { télévision. }\end{array}$ & today. \\
\hline $\begin{array}{l}\text { Finale }(\mathrm{N}, \text { Ref no. } 18 \mathrm{pg} .6) \text { Contexte } \\
\text { source (source context) : Pour la } \\
\text { première fois, la phase finale de la } \\
\text { compétition regroupe vingt-quatre } \\
\text { équipes. }\end{array}$ & $\begin{array}{l}\text { Final (N, Ref no. } 5 \text { pg.14) Contexte source } \\
\text { (source term): Nationals final in which } \\
\text { Egypt beat the elephants in a thrilling penalty } \\
\text { shoot out. }\end{array}$ \\
\hline $\begin{array}{l}\text { Football ( } \underline{N, \text { Ref. no. } 4 \text { pg. } 16}) \text { Contexte } \\
\text { source (source context): Le football } \\
\text { C'est d'abord le plaisir. }\end{array}$ & $\begin{array}{l}\text { Ball }(\mathrm{N}, \text { Ref no. } 5 \text { pg. } 12) \text { Contexte source } \\
\text { (source context): Football is an interesting } \\
\text { game. }\end{array}$ \\
\hline $\begin{array}{l}\text { Footballeurs }(\mathrm{N}, \text { Ref. no.14 pg.10) } \\
\text { Contexte source : (source context). A la } \\
\text { date du } 19 \text { juin, les footballeurs africans y } \\
\text { tiennent les trois premières places. }\end{array}$ & $\begin{array}{l}\text { Footballers (N, Ref no. } 1 \text { pg. } 2) \text { Contexte } \\
\text { Source (source context). Pele, the world's } \\
\text { acclaimed greatest footballer. }\end{array}$ \\
\hline $\begin{array}{l}\text { Finalistes ( } \mathrm{N}, \text { No.7 pg.14) Contexte } \\
\text { source (source context) : Dans ce } \\
\text { contexte les cinq finalistes Africains, } \\
\text { Afrique du sud }\end{array}$ & $\begin{array}{l}\text { Finalists (N, No. } 21 \mathrm{pg} .24) \text { Contexte } \\
\text { d'arrivée (Target context): Under FIFA } \\
\text { expanded World cup format... } 94 \text { to five at } \\
\text { France } 98,32 \text { finalists including hosts } \\
\text { France. }\end{array}$ \\
\hline $\begin{array}{l}\text { Gardiens de but }(\mathrm{N}, \text { Ref. no.12 pg.10) } \\
\text { Contexte source (source context). Il ne } \\
\text { faut pas pour autant innocenter les } \\
\text { gardiens de but. }\end{array}$ & $\begin{array}{l}\text { Goal keeper (N, No. } 11 \text { pg.4) Contexte } \\
\text { d'arrivée (Target context): The guest like } \\
\text { force of his long range pile drivers can leave } \\
\text { any well positioned goal keeper gasping at } \\
\text { thin air. }\end{array}$ \\
\hline $\begin{array}{l}\text { Jeux Olympiques }(\mathrm{N}, \text { No.12 pg.14) } \\
\text { Contexte source (source context). Pour } \\
\text { la première fois, trente-deux équipes } \\
\text { reparties en huit groupe participeront à ce } \\
\text { qui, au fil des années, est devenu le plus } \\
\text { important événement mediatico sportif, } \\
\text { avec les jeux Olympiques. }\end{array}$ & $\begin{array}{l}\text { Olympique games (N, No. } 21 \mathrm{pg} .3 \text { ) } \\
\text { Contexte d'arrivée (Target context)... } \\
\text { although we did it in Atlanta during the } \\
\text { Olympic games. }\end{array}$ \\
\hline $\begin{array}{l}\text { Joueurs ( } \mathrm{N}, \text { no.13 pg.10) Contexte } \\
\text { d'arrivée (Target context) : Première } \\
\text { explication à ces mauvais résultants : la } \\
\text { qualité des joueurs. }\end{array}$ & $\begin{array}{l}\text { players }(\mathrm{N}, \text { no. } 22 \mathrm{pg} .13) \text { Contexte } \\
\text { d'arrivée (Target context): Among the new } \\
\text { players are veteran Shola Oyewusi. }\end{array}$ \\
\hline $\begin{array}{l}\text { Latéraux (N, No.17 pg.8) Contexte } \\
\text { d'arrivée (Target context) : Un joueur } \\
\text { comme David Nyath, considérée comme }\end{array}$ & $\begin{array}{l}\text { Striker (N, no.4 pg.20) Contexte } \\
\text { d'arrivée (Target context)... Yekini, } \\
\text { former Super Eagles Striker and } 1993\end{array}$ \\
\hline
\end{tabular}




\begin{tabular}{|c|c|}
\hline l'un des meilleurs latéraux du monde. & African Footballer of the year. \\
\hline $\begin{array}{l}\text { Légendaire ( } \mathrm{N}, \text { no. } 7 \text { pg. } 23) \text { Contexte } \\
\text { d'arrivée (Target context) : Bien sŭr, le } \\
\text { légendaire Roger Milla n'est plus là. }\end{array}$ & $\begin{array}{l}\text { Legendary ( } \mathrm{N}, \text { no. } 3 \text { pg.18 }) \text { Contexte } \\
\text { d'arrivée (Source context): Dicicco one of } \\
\text { the most legendary coaches in U.S. }\end{array}$ \\
\hline $\begin{array}{l}\text { Lions Indomptables (Indomitable Lions) } \\
\text { (N, no } 18 \text { pg. 20) Contexte source } \\
\text { (source context) : En 1990, le Mondiale } \\
\text { italien, au demeurant très fiable sur le plan } \\
\text { du spectacle, aura été littéralement } \\
\text { illuminé par la permance des Lions } \\
\text { indomptables camerounais. }\end{array}$ & $\begin{array}{l}\text { Indomitable Lion ( } \mathrm{N}, \text { No.13 pg.14) } \\
\text { Contexte d'arrivée: (source context). The } \\
\text { Indomitable Lions were written off after a } 4 \\
-2 \text { defeat. }\end{array}$ \\
\hline $\begin{array}{l}\text { Maître entraîneurs ( } \mathrm{N}, \text { no.18 pg.25) } \\
\text { Contexte Source (Source context) }: \text { Des } \\
\text { maîtres entraîneurs - Marocains, les } \\
\text { Tunisles etc. }\end{array}$ & $\begin{array}{l}\text { Head coach }(\mathrm{N}, \text { no. } 2 \text { pg.14) Contexte } \\
\text { source (source term): Head coach of First } \\
\text { Bank Basketball Club, Adewunmi Aderemi }\end{array}$ \\
\hline $\begin{array}{l}\text { Match }(\underline{N}, \text { no. } 20 \text { pg. } 13) \text { Contexte source } \\
\text { (source context)... d'un match à l'autre } \\
\text { dans la défaite. }\end{array}$ & $\begin{array}{l}\text { Match ( } \mathrm{N}, \text { no. } 2 \text { pg.4) Contexte d'arrivée } \\
\text { (Target context): I believe that the guys } \\
\text { played very well particularly for that match. }\end{array}$ \\
\hline $\begin{array}{l}\text { Médaille d'or (N, no.20 pg.5) Contexte } \\
\text { Source (Source context). Beaucoup en } \\
\text { revanche verraient bien les, Nigérians, } \\
\text { médaille d'or aux derniers jeux } \\
\text { Olympiques. }\end{array}$ & $\begin{array}{l}\text { Gold medal (N, no. } 10 \text { pg.16) Contexte } \\
\text { d'arrivée (Target context): The } 2004 \text { gold } \\
\text { medal at the National Sports Festival Abuja. }\end{array}$ \\
\hline $\begin{array}{l}\text { Milieu de terrain }(\mathrm{N}, \text { no. } 1 \mathrm{pg} .30) \\
\text { Contexte d'arrivée (source context) : Il } \\
\text { devrait devenir un très grand milieu de } \\
\text { terrain. }\end{array}$ & $\begin{array}{l}\text { Mid-fielder ( } \mathrm{N}, \text { no. } 10 \text { pg.17) Contexte } \\
\text { d'arrivée (Target context): They gave us } \\
\text { our toughest game so far said the Ismaili } \\
\text { mid-fielder. }\end{array}$ \\
\hline $\begin{array}{l}\text { Penalty }(\mathrm{N}, \text { no14 pg.27) Contexte source } \\
\text { (source context) : Les ralentis seront } \\
\text { surtout utilisés pour les fautes entraînant } \\
\text { un penalty. }\end{array}$ & $\begin{array}{l}\text { Penalty (N, no. } 9 \text { pg.14) Contexte } \\
\text { d'arrivée (Target context): In } 1992 \text { before } \\
\text { bowing out on penalities to Cote d'Ivoire... }\end{array}$ \\
\hline $\begin{array}{l}\text { Première mi -temps }(\mathrm{N}, \text { no.13 pg.15) } \\
\text { Contexte Source (Source context) : } \\
\text { Maldini pour qu'ils ouvrent le score en } \\
\text { milieu de première mi-temps. }\end{array}$ & $\begin{array}{l}\text { First half }(\underline{N}, \text { no. } 9 \text { pg. } 13) \text { Contexte } \\
\text { d'arrivée (Target context): Kader Keita's } \\
\text { goal in the first half keeping them down... }\end{array}$ \\
\hline $\begin{array}{l}\text { Prolongation ( } \mathrm{N}, \text { no. } 19 \text { pg. } 14) \text { Contexte } \\
\text { source (source context) : Deux buts dont } \\
\text { I'un pendant la prolongation... }\end{array}$ & $\begin{array}{l}\text { Extra time (N, no. } 11 \mathrm{pg} .24) \text { Contexte } \\
\text { d'arrivée (Target context): The two teams } \\
\text { were given } 15 \text { minutes extra time each to } \\
\text { determine who will finally go with the }\end{array}$ \\
\hline
\end{tabular}




\begin{tabular}{|c|c|}
\hline & trophy. \\
\hline $\begin{array}{l}\text { Quarts de finales }(\underline{\mathrm{N}, \text { no.12 pg. } 31)}) \\
\text { Contexte source (source context) : } \\
\text { Hristo Stoichkov }(0-3) \text { Futurs tombeurs de } \\
\text { l'Allemagne en quarts de finale... }\end{array}$ & $\begin{array}{l}\text { Quarter final ( } \mathrm{N}, \text { no. } 21 \mathrm{pg} .32) \text { Contexte } \\
\text { d'arrivée (Target context). There is no place } \\
\text { in the Bulgarian squad for Yordan Lechkov, } \\
\text { who headed the quarter-final. }\end{array}$ \\
\hline $\begin{array}{l}\text { spectateurs }(\mathrm{N}, \text { no. } 12 \text { pg.20) Contexte } \\
\text { source (source context) : La cérémonie } \\
\text { d'ouverture notamment suivle par trente- } \\
\text { cinq mille spectateurs... }\end{array}$ & $\begin{array}{l}\text { spectators }(\mathrm{N}, \text { no. } 22 \mathrm{pg} .13) \text { Contexte } \\
\text { source (source context): The spectators } \\
\text { aligned themselves once more. }\end{array}$ \\
\hline $\begin{array}{l}\text { Supporters }(\underline{\mathrm{N}, \text { no. } 13 \mathrm{pg} .27)} \text { Contexte } \\
\text { source (soruce context) : Ils n'ont pas } \\
\text { totalement rassuré leurs supporters. }\end{array}$ & $\begin{array}{l}\text { Supporters }(\mathrm{N}, \text { no. } 10 \mathrm{pg} .19) \text { Contexte } \\
\text { d'arrivée (Target context): The black stars } \\
\text { now have a chance because of the home } \\
\text { supporters. }\end{array}$ \\
\hline $\begin{array}{l}\text { Télévisions }(\mathrm{N}, \text { no. } 14 \mathrm{pg} .4 \text { ) Contexte } \\
\text { source (source context). Télévision } \\
\text { firmes d'articles de sport publicitaires. }\end{array}$ & $\begin{array}{l}\text { Television ( } \mathrm{N}, \text { no. } 21 \mathrm{pg} .15) \text { Contexte } \\
\text { d'arrivée (Target context): The streets } \\
\text { sought the nearest available television. }\end{array}$ \\
\hline $\begin{array}{l}\text { Trophée ( } \mathrm{N}, \text { no.19 pg.19) Contexte } \\
\text { source (source context) }: \text { Les étalons qui } \\
\text { lors de leurs deux précédentes } \\
\text { participation (1978 et 1996) avaient été } \\
\text { rapidement élimines, ne rêvent pas eux de } \\
\text { remporter le trophée. }\end{array}$ & $\begin{array}{l}\text { Trophy (N, no. } 8 \text { pg.24) Contexte } \\
\text { d'arrivée (Target context). At stake is } \\
\text { football's ultimate prime a glittering trophy. }\end{array}$ \\
\hline $\begin{array}{l}\text { Tournoi (N, no.12 pg.10) Contexte } \\
\text { d'arrivée (Target context). Le tournoi } \\
\text { olympique d'Atlanta deux ans plus tard } \\
\text { constituent autant de resultants probants } \\
\text { qui ont transforme l'état d'esprit des } \\
\text { footballeurs de l'élite. }\end{array}$ & $\begin{array}{l}\text { Tournament }(\mathrm{N}, \text { no. } 8 \text { pg. } 15) \text { Contexte } \\
\text { d'arrivée (Target context): This tournament } \\
\text { was supposed to make up for the } 2006 \text { World } \\
\text { cup in which we failed to participate. }\end{array}$ \\
\hline $\begin{array}{l}\text { Vainquers }(\mathrm{N}, \text { no.17 pg.15) Contexte } \\
\text { source (source context). Face aux } \\
\text { vainquers de la coupe d'Afrique. }\end{array}$ & $\begin{array}{l}\text { winners ( } \mathrm{N}, \text { no. } 1 \text { pg. } 10 \text { ) Contexte } \\
\text { d'arrivée (Target context): Here are the } \\
\text { winners of the Nation's cup. }\end{array}$ \\
\hline $\begin{array}{l}\text { Victoire }(\mathrm{N}, \text { no.17 pg.12) } \text { Contexte } \\
\text { source (source context) : Une première } \\
\text { Victoire stupéfiante sur les Argentins de } \\
\text { Diego. }\end{array}$ & $\begin{array}{l}\text { Victory (N, no. } 3 \text { pg.24) Contexte } \\
\text { d'arrivée (Target context). Cameroun into } \\
\text { the quarter-finals with an easy } 3-0 \text { victory. }\end{array}$ \\
\hline
\end{tabular}




\section{Analyse des Termes}

* Le terme world cup est terme d'arrivée. Il est employé dans le contexte (Champion, Friday June 19, 1998) "History is replete with stars who came to the World cup". En parlant du terme source Coupe du monde dans le contexte source, on cite (Jeune Afrique No 1952, Du 10 juin au 12 juillet 1998) «le plus écart de faute dans l'histoire de la coupe du monde «.Dans les termes sources et d'arrivée world cup et coupe du monde représente le championnat de football au niveau international.

* Le terme corner est terme source.Il est employé dans le contexte source (Afrique Noire No, 1990 Du 2 au ) « Les corners par exemple seront filmés avec une camera haute. D'autre part le terme d'arrivée corner est employé ainsi dans le contexte d'arrivée (Champion, Saturday June 13, 1998). «Soon after, the Nigerian team settled down to business forcing the first corner in eighth minute".Corners et corner représentent la sortie du ballon du terrain.

* Le terme demi- finales est terme source utilisé dans le contexte source (Jeune Afrique No. 1946, Du 28 Avril au 4 mai 1998.) » Les demi - finales, le match de classement et naturellement, la finale...» D'autre part, semi finals est terme d' arrivée employé dans le contexte d'arrivée (Champion, Sunday June 14, 1998) «Egypt meet Ivory Coast in semi - finals tomorrow. » Demi-finale et semi-finals représentent une étape dans un championnat.

* Le terme source début de match est employé dans le contexte source (Jeune Afrique No. 1990, Du 2 au 8 mars 1999). "Le début de match des lions est catastrophe".Pour le terme d'arrivée opening match dans le contexte d'arrivée on cite (Champion, Friday June 5, 1998)

«Super Eagles opening match against Bulgaria for years ago in USA."Debut de match et opening match représente le commencement d'un match.

* Le terme source défense est employé dans le contexte source (Jeune Afrique No. 1946, Du 28 Avril au 4 mai 1998.) « En d'autres termes, il est l'avantgarde de sa défense et l'arrière - garde de son attaque » Le terme d'arrivée defence est employé dans (Vanguard, Wednesday February 6, 2008). le contexte d'arrivée "... Europe's best sweeper to marshal the defence". Défense et defence représentent la position des joueurs juste devant le Poteau de but.

* Le terme source élimination est utilisé dans contexte source dans (Jeune Afrique No. 1952, Du 9 juin au 12 juillet 1998.) «... Une élimination sans 
gloire ». D'autre part, le terme d'arrivée elimination est employé dans le contexte d'arrivée dans (Champion, Friday June 5, 1998)

Ainsi "Nigeria's elimination at the quarter final..."Elimination et elimination représentent le font d'être battu.

\section{La conclusion}

Pour faire ce travail nous avons utilisé les différents journaux en anglais et en français qui traitaient le football pendant le coupe du monde ou coupe africaine. Nous voulons réveiller l'intérêt du traducteur dans ce domaine. Nous voulons que d'autres collègues continuent à faire leurs travaux sur ce domaine de la linguistique car la terminologie est vaste.

Nous avons aussi montré qu'un terme n'a qu' un sens.

\section{Références}

Afrique Noire No, 1990 Du 2 au 8 mars 1999

Champion, Sunday June 7, 1998.

Champion, Sunday June 14, 1998

Champion, Monday June 1, 1998.

Champion, Wednesday June 10, 1998

Champion, Friday June 5, 1998

Champion, Friday June 19, 1998.

Champion, Saturday June 6, 1998.

Champion, Saturday June 13, 1998.

Daily Champion, Wednesday June 10, 1998.

Daily Independent, Tuesday February 5, 2006

Jeune Afrique No. 1936, 17 au 23 fevrier 1998.

Jeune Afrique No. 1937, Du 24 fevrier au 2 mars 1998.

Jeune Afrique No. 1946, Du 28 Avril au 4 mai 1998.

Jeune Afrique No. 1952, Du 9 juin au 12 juillet 1998.

Jeune Afrique No. 1953, 16 au 22 juin 1998.

Jeune Afrique No. 1954, 23 au 29 juin 1998. 
Jeune Afrique No 1952, Du 10 juin au 12 juillet 1998.

Jeune Afrique No. 1990, Du 2 au 8 mars 1999.

Jeune Afrique No. 1952, 9 au 15 juin 1999.

The Guardian, Wednesday February 6, 2008.

The Guardian, Thursday February 7, 2008.

Vanguard, Wednesday February 6, 2008.

Vanguard, Saturday February 2, 2008.

Johansson S. et Hofland K.. (1994). "Towards an English-Norwegian Parallel Corpus" in Fries, G. Tothe \& P. Scheidner (eds.) Creating and Using English Language Corpora.Amsterdam and Atlanta, G. A. Rodopi, 225. 